\title{
HOCHSCHILD AND CYCLIC HOMOLOGY ARE FAR FROM BEING HOMOTOPY FUNCTORS
}

\author{
S. GELLER AND C. WEIBEL
}

(Communicated by Donald S. Passman)

\begin{abstract}
Given a homology theory $H_{*}(A)$ on rings, based on a natural chain complex, one can form a new theory $H_{*}^{h}(A)$ which is universal with respect to the homotopy property $H_{*}(A) \simeq H_{*}(A[t])$. We show that the homotopy theories $H H_{*}^{h}$ and $H C_{*}^{h}$ associated to Hochschild and cyclic homology are both zero. On the other hand, if $H C^{-}$denotes Goodwillie's variant of cyclic homology, and $A$ contains a field of characteristic 0 , we show that $\left(H C^{-}\right)_{*}^{h} A$ is Connes' periodic cyclic homology $H P_{*}(A)$.
\end{abstract}

A functor $F$ from the category of associative rings with unit to an abelian category is called a homotopy functor if $F(A) \rightarrow F(A[t])$ is an isomorphism for every ring $A$. If $[F] A$ denotes the coequalizer of

$$
F(A[t]) \underset{t=1}{\stackrel{t=0}{\rightrightarrows}} F(A),
$$

then $[F]$ is a homotopy functor, and $F \rightarrow[F]$ is universal with respect to maps from $F$ to homotopy functors.

One way to measure how far $F$ is from a homotopy functor is to study the groups $N^{n} F(A)$ of [Bass, XII], defined by

$$
N^{n} F(A)=\bigcap_{i=1}^{n} \operatorname{ker}\left\{F\left(A\left[t_{1}, \ldots, t_{n}\right]\right) \stackrel{t_{i}=0}{\rightarrow} F\left(A\left[t_{1}, \ldots, \hat{t}_{i}, \ldots, t_{n}\right]\right)\right\} .
$$

The map $A\left[t_{1}, \ldots, t_{n}\right] \rightarrow A\left[t_{1}, \ldots, t_{n-1}\right]$ sending $t_{n}$ to $1-\sum t_{j}$ induces a map $d_{0}: N^{n} F(A) \rightarrow N^{n-1} F(A)$, and these fit together to form a chain complex

$$
N^{*} F(A): 0 \leftarrow F(A) \stackrel{t=1}{\longleftarrow} N F(A) \stackrel{d_{0}}{上} N^{2} F(A) \stackrel{d_{0}}{\longleftarrow} N^{3} F(A) \leftarrow \cdots .
$$

Received by the editors August 9, 1988 .

1980 Mathematics Subject Classification (1985 Revision). Primary 16A61; Secondary 18G30, $19 \mathrm{D} 55$.

Key words and phrases. Hochschild homology, cyclic homology, homotopy functor, Moore complex.

The first author was supported by National Science Foundation Grant RII-8700009.

The second author was partially supported by National Science Foundation Grants DMS-8503018 and DMS-8803497. 
We call $N^{*} F(A)$ the "Moore Complex" of $F(A)$. In $\S 2$, we show that, if $A$ is a $k$-algebra, $H H_{m}(A)$ denotes Hochschild homology, and $\Omega_{A / k}^{m}$ denotes Kähler differentials (for commutative $A$ ), then $N^{*} H H_{m}(A)$ and $N^{*} \Omega_{A / k}^{m}$ are exact. The corresponding result for the cyclic homology group $H C_{m}$ is not true for $m \neq 0$. (See $\S 3$.)

When $F_{*}(A)$ arises as the homology of a natural chain complex $C(A)$, as is the case for Hochschild and cyclic homology, there is a more subtle notion of homotopy. Following [W1,2.2], we define a new homotopy functor $F_{*}^{h}$ in $\S 1$, arising as the homology of the total chain complex of $N^{\circ} C(A)$, which is universal in the appropriate sense. We then show that $H H_{*}^{h}(A)=H C_{*}^{h}(A)=$ 0 , which shows just how far Hochschild and cyclic homology are from being homotopy functors. Finally, we apply these ideas in $\S 4$ to Goodwillie's variant $H C_{*}^{-}$of cyclic homology. If $A$ contains $\mathbf{Q}$, we show that $\left(H C^{-}\right)_{*}^{h}(A)$ is $H P_{*}^{*}(A)$, Connes' periodic cyclic homology. This emphasizes the central role of $H C_{*}^{-}$in cyclic homology.

\section{The MOORE COMPLEX}

In this section, we discuss ways of measuring how much a functor $F$ fails to be a homotopy functor. We use the simplicial ring $\Delta A$ of $A$-valued algebraic functions on the standard $p$-simplex (denoted $P(A)$ in [And]). Thus,

$$
\Delta_{n} A=A\left[t_{0}, \ldots, t_{n}\right] /\left(\sum t_{j}=1\right) \simeq A\left[t_{1}, \ldots, t_{n}\right],
$$

while the face and degeneracy maps are given by

$$
\begin{aligned}
& d_{i}\left(t_{i}\right)=0, \quad s_{i}\left(t_{i}\right)=t_{i}+t_{i+1}, \\
& d_{i}\left(t_{j}\right)=t_{j} \text { or } t_{j-1}, \quad \text { and } s_{i}\left(t_{j}\right)=t_{j} \text { or } t_{j+1}
\end{aligned}
$$

if $i \neq j$, depending on whether $j<i$ or $j>i$.

Now let $F$ be a functor from rings to an abelian category. It is well-known [May, §22] that the simplicial homotopy groups of the simplicial object $F(\Delta A)$ are the same as the homology groups of either the chain complex

$$
0 \leftarrow F(A) \stackrel{d_{0}-d_{1}}{\leftarrow} F\left(A\left[t_{1}\right]\right) \leftarrow \ldots \stackrel{\sum(-1)^{i} d_{i}}{\leftarrow} F\left(A\left[t_{1}, \ldots, t_{n}\right]\right) \leftarrow \ldots
$$

or of the associated "Moore complex" $N^{*} F(A)$ described in the introduction. For example, it is easy to see that

$$
\pi_{0} F(\Delta A)=H_{0}\left(N^{*} F(A)\right)=[F] A .
$$

We may think of the rest of the $\pi_{i} F(\Delta A)=H_{i}\left(N^{*} F(A)\right)$ as left derived functors of $[F]$.

Now suppose that we are given a functorial chain complex $C(A)$, and set $F_{n}(A)=H_{n}(C(A))$. In addition to the homotopy functors $\left[F_{n}\right]$, we have the functors $F_{n}^{h}(A)=H_{n}(|C(\Delta A)|)$, where $|C(\Delta A)|$ means the total complex of the associated double complex $N^{*} C(A)$. As in [W1, 2.4], the functors $F_{n}^{h}(A)$ are 
homotopy functors. If $C$ is bounded below, the maps $\left[F_{n}\right] \rightarrow F_{n}^{h}$ are the edge maps of a convergent spectral sequence

$$
E_{p q}^{2}=H_{p}\left(N^{*} F_{q}(A)\right) \Rightarrow F_{p+q}^{h}(A) .
$$

(Cf. [CE, XV.6], [And, 2.3].) We draw attention to an immediate consequence of this formalism:

Lemma 1.2. Suppose $F_{*}(A)=H_{*}(C(A))$ for a natural complex $C(A)$.

(i) If $F_{m}(A)=F_{m}\left(A\left[t_{1}, \ldots, t_{n}\right]\right)$ for all $m, n$, then $F_{m}(A)=F_{m}^{h}(A)$ for all $m$.

(ii) If the Moore complexes $N^{\circ} F_{m}(A)$ are exact for all $m$, then $F_{m}^{h}(A)=0$ for all $m$.

\section{HoCHSCHILD HOMOLOGY}

The formalism of the last section makes sense if we replace the category of rings by a category $\mathscr{R}$ such that, whenever $A$ is in $\mathscr{R}$, so are the objects and maps in $\Delta A$. For example if $k$ is a commutative ring, we can consider either the category of $k$-algebras and the Hochschild homology functors $H_{m}(A)$, or the category of commutative $k$-algebras and the Kähler differential functors $\Omega_{A / k}^{m}$. Note that $H H_{m}(A)$ is $H_{m}$ of the natural chain complex of $k$-modules

$$
C(A): 0 \leftarrow A \leftarrow A \otimes_{k} A \leftarrow A \otimes_{k} A \otimes_{k} A \leftarrow \ldots
$$

(see [CE, IX.6], [Mac, X.4]). We can now construct the functors $H H_{m}^{h}$ as in $\S 1$.

Theorem 2.1. For every $m$ and every k-algebra $A$,

(i) $H H_{m}^{h}(A)=\left[H H_{m}\right] A=0$.

(ii) The Moore complex $N^{*} H H_{m}(A)$ is exact, where $N^{*} H H_{m}(A)$ is:

$$
0 \leftarrow H H_{m}(A) \leftarrow N H H_{m}(A) \leftarrow N^{2} H H_{m}(A) \leftarrow \cdots .
$$

Theorem 2.2. For every $m$, let $\Omega^{m}(A)=\Omega_{A / k}^{m}$ denote the Kähler differential functor. Then for every commutative ring $A$ the Moore complex

$$
N^{*} \Omega^{m}(A): 0 \leftarrow \Omega^{m}(A) \leftarrow N \Omega^{m}(A) \leftarrow N^{2} \Omega^{m}(A) \leftarrow \ldots
$$

is split exact. (The splitting is not natural.)

Proof that Theorem 2.2 implies Theorem 2.1. The Künneth formula [Mac, V.10.1 and X.7] yields

$$
H H_{m}\left(A\left[t_{1}, \ldots, t_{n}\right] \simeq \bigoplus_{i+j=m} H H_{i}(A) \bigotimes H H_{j}\left(k\left[t_{1}, \ldots, t_{n}\right]\right),\right.
$$

so that $N^{n} H H_{m}(A) \simeq \bigoplus H H_{i}(A) \otimes N^{n} H H_{j}(k)$. Thus it is enough to show that $N^{*} H H_{j}(k)$ is split exact for all $j$. But this is Theorem 2.2, since by [HKR, 5.1] we have

$$
H H_{j}\left(k\left[t_{1}, \ldots, t_{n}\right]\right) \simeq \Omega_{k\left[t_{1}, \ldots, t_{n}\right] / k}^{j} .
$$


Proof of Theorem 2.2. For every commutative $k$-algebra $A$,

$$
\Omega_{A\left[t_{1}, \ldots, t_{n}\right] / k} \simeq\left(\Omega_{A / k} \bigotimes_{A} A\left[t_{1}, \ldots, t_{n}\right]\right) \bigoplus\left(A \bigotimes \Omega_{k\left[t_{1}, \ldots, t_{n}\right] / k}\right)
$$

by [Mat, 26.J], so that

$$
\Omega^{m}\left(A\left[t_{1}, \ldots, t_{n}\right]\right)=\bigoplus_{i+j=m} \boldsymbol{\Omega}^{i}(A) \bigotimes \Omega^{j}\left(k\left[t_{1}, \ldots, t_{n}\right]\right) .
$$

Therefore it is enough to prove that $N^{*} \Omega^{m}(k)$ is split exact for all $m$. But each $k$-module $N^{p} \Omega^{m}(k)$ is projective, since by [Bass, XII.7.3] it is a direct summand of the free $k$-module $\Omega^{m}\left(k\left[t_{1}, \ldots, t_{n}\right]\right)$. Therefore it suffices to prove that $N^{*} \Omega^{m}(k)$ is exact for all $m$.

We proceed by induction on $m$. When $m=0, \Omega^{0}(A)=A$, and $N^{p} \Omega^{0}(k)$ is the ideal $\left(t_{1} \ldots t_{n}\right) k\left[t_{1}, \ldots, t_{n}\right]$ of the ring $k\left[t_{1}, \ldots, t_{n}\right]$. The differential $N^{n} \Omega^{0}(k) \rightarrow N^{n-1} \Omega^{0}(k)$ sends $t_{j}$ to $t_{j}$ for $j \neq n$, and sends $t_{n}$ to $1-\sum_{j=1}^{n-1} t_{j}$. The proof of exactness of $N^{*} \Omega^{0}(k)$ is now a pleasant exercise.

Let us analyze the groups $N^{m} \Omega^{m}(k)$ slightly, introducing some notation, before continuing. We write $k\left[T_{n}\right]$ for $k\left[t_{1}, \ldots, t_{n}\right]$. Since $\Omega^{m}\left(k\left[T_{n}\right]\right)=0$ for $m>n, N^{n} \Omega^{m}(k)=0$ for $n<m$. Thus the complex $N^{*} \Omega^{m}(k)$ starts in degree $m$ with

$$
N^{m} \Omega^{m}(k)=\Omega^{m}\left(k\left[T_{m}\right]\right) \simeq k\left[T_{m}\right] \quad \text { on } d t_{1} \wedge \cdots \wedge d t_{m} .
$$

In order to describe the rest of $N^{*} \Omega^{m}(k)$, let $I=\left\{i_{1}, \ldots, i_{m}\right\}$ be an ordered $m$-element subset of $\{1, \ldots, n\}$ ('ordered' means $i_{1}<\cdots<i_{m}$ ). Set

$$
\omega_{n}^{m}(I)=\left(\prod_{j \notin I} t_{j}\right) d t_{i_{1}} \wedge \cdots \wedge d t_{i_{m}} \quad \text { in } N^{n} \Omega^{m}(k) .
$$

Then a straightforward computation shows that $N^{n} \Omega^{m}(k)$ is the direct sum of copies of $k\left[T_{n}\right]$ on generators $\omega_{n}^{m}(I)$.

In order to take advantage of the inductive hypothesis, we filter the complex $N^{*} \Omega^{m}(k)$ by subcomplexes $F_{p}{ }^{\cdot}$, where $F_{p}^{n}$ is the $k\left[T_{n}\right]$-submodule of $N^{n} \Omega^{m}(k)$ generated by those $\omega_{n}^{m}(I)$ with $I \subseteq\{1, \ldots, p\}$. Since $N^{*} \Omega^{m}(k)$ is the union of the $F_{p}^{\cdot}$, there is a convergent spectral sequence

$$
E_{p q}^{1}=H_{p+q}\left(F_{p}^{\cdot} / F_{p+1}^{\cdot}\right) \Rightarrow H_{p+q}\left(N^{\bullet} \Omega^{m}(k)\right)
$$

attached to the filtration [Mac, XI.3.1 and 3.2]. Since $F_{p}^{p+q}=0$ for $q<0$ or $p<m$, this is a first quadrant spectral sequence. To prove Theorem 2.2, we will show that $E_{p q}^{2}=0$ for all $p, q$.

We first show that $E_{p q}^{1}=0$ for $q \neq 0$. Since $F_{m-1}^{\cdot}=0, E_{p q}^{1}=0$ for $p<m$, and we may begin with $E_{m q}^{1}$. Let $\omega_{r}=\omega_{r}^{m}(1,2, \ldots, m)$; the complex $F_{m}^{\cdot}$ has $F_{m}^{r}=0$ for $r<m$, and $F_{m}^{r} \cong k\left[T_{r}\right]$ on generator $\omega_{r}$. Since $d^{1}: F_{m}^{r+1} \rightarrow F_{m}^{r}$ sends $\omega_{r+1}$ to $\left(1-\sum t_{j}\right) \omega_{r}$, we can identify $F_{m}^{*}$ with that portion of the exact 
complex $N^{*} \Omega^{0}(k)$ which lies in degrees $\geq m$. Hence $E_{m q}^{1}=H_{m+q}\left(F_{m}^{\cdot}\right)=0$ for $q \neq 0$, and

$$
E_{m 0}^{1}=H_{m}\left(F_{m}^{\cdot}\right)=\left(k\left[T_{m}\right] /\left(1-\sum t_{j}\right) k\left[T_{m}\right]\right) \omega_{m} \cong k\left[T_{m-1}\right] .
$$

When $p>m$, the computation of $E_{p q}^{1}$ is slightly more complicated, because $F_{p}^{r} / F_{p-1}^{r} \cong \oplus_{I} k\left[T_{r}\right] \omega_{r}^{m}(I \cup\{p\})$, the sum running over all ordered $(m-1)$ tuples $I$ of $\{1, \ldots, p-1\}$. The boundary map $d^{1}$ sends $\omega_{r}^{m}(I \cup\{p\})$ to $\left(1-\sum t_{j}\right) \omega_{r-1}^{m}(I \cup\{p\})$ if $r>p$. (If $r \leq p, d^{1}=0$ because $F_{p}^{r-1}=0$.) Thus $F_{m}$ is a direct sum (over $I$ ) of subcomplexes, each isomorphic to that portion of the exact complex $N^{*} \Omega^{0}(k)$ lying in degrees $\geq p$. In this case $E_{p q}^{1}=H_{p+q}\left(F_{p}^{\cdot} / F_{p-1}^{\cdot}\right)=0$ if $q \neq 0$, and

$$
\begin{aligned}
E_{p 0}^{1} & =H_{p}\left(F_{p}^{\cdot} / F_{p-1}^{\cdot}\right)=\bigoplus_{I}\left(k\left[T_{p}\right] /\left(1-\sum t_{i}\right) k\left[T_{p}\right]\right) \omega_{p}^{m}(I \cup\{p\}) \\
& \cong \bigoplus_{I} k\left[T_{p-1}\right] \omega_{p-1}^{m-1}(I) d t_{p} .
\end{aligned}
$$

Having shown that $E_{p q}^{1}=0$ for $q \neq 0$, we need only see that the complex $E_{* 0}^{1}$ is exact in order to see that $E_{p q}^{2}=0$ for all $p, q$, and hence that $N^{*} \Omega^{m}(k)$ is exact. But exactness of $E_{* 0}^{1}$ follows from our inductive hypothesis and

Lemma 2.3. For each $m \geq 1$, the chain complex $E_{* 0}^{1}$ is naturally isomorphic to the chain complex $N^{*} \Omega^{m-1}(k)[-1]$, i.e., $N^{*} \Omega^{m-1}(k)$ shifted to the left by one.

Proof. Letting $I$ run over all ordered $(m-1)$-tuples of $\{1, \ldots, p-1\}$, and writing $\omega(I)$ for $\omega_{p-1}^{m-1}(I)$, we have

$$
\begin{aligned}
& N^{p-1} \Omega^{m-1}(k)=\bigoplus_{I} k\left[T_{p-1}\right] \omega(I), \\
& E_{p 0}^{1} \cong \bigoplus_{I^{k}}^{k}\left[T_{p-1}\right] \omega(I) \wedge d t_{p} .
\end{aligned}
$$

Thinking of $k\left[T_{p-1}\right] \omega(I) \wedge d t_{p}$ as a subgroup of $F_{p}^{p} \subseteq \Omega^{m}\left(k\left[T_{p}\right]\right)$, we can calculate $d^{1}$ by applying the differential $d_{0}: N^{p} \Omega^{m}(k) \rightarrow N^{p-1} \Omega^{m}(k)$ in the original Moore complex. If $f \in k\left[T_{p-1}\right]$, we have

$$
\begin{aligned}
d^{1}\left(f \omega(I) \wedge d t_{p}\right)= & -f \omega(I) \wedge \sum_{j=1}^{p-1} d t_{j} \quad \bmod \left(F_{p-2}+d\left(F_{p-1}^{p}\right)\right) \\
& \left\{\begin{array}{c}
-\bar{f}\left(1-\sum t_{j}\right) \omega_{p-2}^{m-1}(I) \wedge d t_{p-1} \\
\text { if } p-1 \notin I, \\
+\bar{f} \omega_{p-2}^{m-2}(I-\{p-1\}) \wedge \sum d t_{j} \wedge d t_{p-1} \\
\text { if } p-1 \in I,
\end{array}\right.
\end{aligned}
$$


where $\bar{f}$ denotes $f\left(t_{1}, \ldots, t_{p-2}, 1-\sum t_{j}\right) \in k\left[T_{p-2}\right]$. Since this is $(-1)$ times the differential of $f \omega(I) \in N^{p-1} \Omega^{m-1}(k)$, it follows that the map from $N^{p-1} \Omega^{m-1}(k)$ to $E_{p 0}^{1}$ sending $f \omega(I)$ to $(-1)^{p} f \omega(I) \wedge d t_{p}$ is an isomorphism of chain complexes, whence the lemma.

Corollary 2.4. If $I$ is an ideal of a $k$-algebra $A$, the relative Moore complex $N^{*} H H_{m}(A, I)$ is split exact for all $m$. If $A \rightarrow B$ sends $I$ isomorphically onto an ideal of the $k$-algebra $B$, then the double relative Moore complex $N^{*} H H_{m}(A, B, I)$ is also split exact for all $m$.

Proof. As in the proof of Theorem 2.1, the Künneth formula yields

$$
H H_{m}(A[T], I[T]) \cong \bigoplus_{i+j=m} H H_{i}(A, I) \bigotimes H H_{j}(k[T]) .
$$

The complex $N^{*} H H_{m}(A, I)$ is therefore the direct sum of the complexes $H H_{i}(A, I) \otimes N^{*} H H_{j}(k)$, each of which is split exact. A similar argument applies in the double relative case.

\section{CyCLIC HOMOLOGY}

We can now apply the results for Hochschild homology to get similar, but weaker, results for cyclic homology. For example, the complex $N^{*} H C_{0}(A)$ is exact for every $k$-algebra $A$ because $H C_{0}=H H_{0}$. On the other hand, since $H C_{2}(k) \cong H C_{2}(k[t]) \cong k$, it is easy to see that $N^{*} H C_{2}(k)$ is not exact.

Recall from $[L Q]$ that there is a double complex $B(A)$ for which $H C_{*}(A)$ is the homology of $\operatorname{Tot}(B(A))$. We can now construct the functors $H C_{m}^{k}$ as in $\S 1$.

Theorem 3.1. For every $k$-algebra $A$,

(i) The Moore complex $N^{*} H C_{0}(A)$ is exact.

(ii) The Moore complex

$$
0 \leftarrow H C_{1}(A) \leftarrow N H C_{1}(A) \leftarrow N^{2} H C_{1}(A) \leftarrow N^{3} H C_{1}(A)
$$

is exact at $H C_{1}(A)$ and $N H C_{1}(A)$, but is not always exact at $N^{2} H C_{1}(A)$.

(iii) $H C_{m}^{h}(A)=0$ for every $m$.

Proof. As in [LQ, 1.6], one has an exact sequence of simplicial complexes

$$
0 \rightarrow C(\Delta A) \rightarrow B(\Delta A) \rightarrow B(\Delta A)[-2] \rightarrow 0 .
$$

This gives a long exact sequence, part of which is

$$
H H_{m}^{h}(A) \rightarrow H C_{m}^{h}(A) \rightarrow H C_{m-2}^{h}(A) \rightarrow H H_{m-1}^{h}(A) .
$$

By Theorem 2.1, this makes the groups $H C_{m}^{h}(A)$ periodic in $m$. However, by construction we have $H C_{m}^{h}(A)=0$ for $m<0$, proving iii). 
To prove ii), we use the spectral sequence

$$
E_{p q}^{1}=N^{p} H C_{q}(A) \Rightarrow H C_{p+q}^{h}(A)=0 .
$$

Now $H C_{q}(A)=0$ for $q<0$, and the row $q=0$ is exact by i). The row $q=1$ is the Moore complex of ii), and we have $E_{01}^{2}=E_{11}^{2}=0, E_{21}^{2} \cong E_{02}^{2}=\left[H C_{2}\right] A$. This proves ii) since, for example, $\left[H C_{2}\right] k=H C_{2}(k) \simeq k$.

Remark 3.2. When $\mathbf{Q} \subset A$, it follows from [G, II.4.6] that

$$
0 \rightarrow N^{p} H C_{m-1}(A) \rightarrow N^{p} H H_{m}(A) \rightarrow N^{p} H C_{m}(A) \rightarrow 0
$$

is exact for $p \geq 1$. Using 3.1(i), we see that the Moore complex $N^{*} H C_{1}(A)$ is exact except at $N^{2} H C_{1}(A)$. Moreover,

$$
H C_{0}(A) \stackrel{S}{\leftarrow} H C_{2}(A) \leftarrow N H C_{2}(A) \leftarrow N^{2} H C_{2}(A)
$$

is exact, $\left[H C_{m}\right] A$ is the image of $S: H C_{m}(A) \rightarrow H C_{m-2}(A)$, and $N^{*} H C_{m}(A)$ is exact at $N^{p} H C_{m}(A)$ for all $p>m$.

Remark 3.3. The proof of Theorem 3.1 makes it clear that, if one cites Corollary 2.4 instead of 2.1 , both 3.1 and 3.2 remain valid with $H C_{m}(A)$ replaced by $H C_{m}(A, I)$ or even $H C_{m}(A, B, I)$. We content ourselves with an application to double relative $K$-theory that we shall need in [GW2].

Corollary 3.4. Let $A \rightarrow B$ be a ring map, and $I$ an ideal of $A$ mapped isomorphically onto an ideal of $B$. Then the following are exact sequences:

(i) the entire Moore complex for $K_{1}(A, B, I)$ :

$$
0 \leftarrow K_{1}(A, B, I) \leftarrow N K_{1}(A, B, I) \leftarrow \cdots \leftarrow N^{p} K_{1}(A, B, I) \leftarrow \cdots .
$$

(ii) The beginning of the Moore complex for $K_{2}(A, B, I)$ :

$$
0 \leftarrow K_{2}(A, B, I) \leftarrow N K_{2}(A, B, I) \leftarrow N^{2} K_{2}(A, B, I) .
$$

Proof. The calculations of [GW1] show that $K_{1}(A, B, I)$ is naturally isomorphic to $H C_{0}(A, B, I)$, so $\left.i\right)$ follows from 3.3. Using the Gersten-Anderson spectral sequence $[\mathrm{W} 3,3.5]$

$$
E_{p q}^{1}=N^{p} K_{q}(A, B, I) \Rightarrow 0 \quad(p \geq 0, q \geq 1) .
$$

We see by i) that $E_{p 1}^{2}=0$ for all $p$. Therefore $E_{02}^{2}=E_{12}^{2}=0$, proving ii).

\section{PeRIODIC CYCLIC HOMOLOGY}

In a similar spirit, one can study the theories $H C_{*}^{-}(A)$ and $H P_{*}(A)$ of [G2]. These functors are the homology groups of natural double chain complexes $B^{-}(A)$ and $B P(A)$, respectively. 
Theorem 4.1. Let $A$ be a $k$-algebra. Then for all $m$,

(i) $\left(H C^{-}\right)_{m}^{h}(A) \cong H P_{m}^{h}(A)$

(ii) There is a natural periodicity isomorphism

$$
S: H P_{m}^{h}(A) \rightarrow H P_{m-2}^{h}(A)
$$

(iii) If $\mathbf{Q} \subset A$, then $H P_{m}(A) \cong H P_{m}^{h}(A)$, and thus

$$
\left(H C^{-}\right)_{m}^{h}(A) \cong H P_{m}(A) \text {. }
$$

Proof. As in [G2], there is a short exact sequence

$$
0 \rightarrow \operatorname{Tot} B^{-}(A) \rightarrow \operatorname{Tot} B P(A) \rightarrow \operatorname{Tot} B(A)[-2] \rightarrow 0 \text {. }
$$

Replacing $A$ with $\Delta A$, we obtain a short exact sequence of simplicial chain complexes, and the corresponding long exact homology sequence is

$$
\cdots H C_{m-1}^{h}(A) \rightarrow\left(H C^{-}\right)_{m}^{h}(A) \rightarrow(H P)_{m}^{h}(A) \rightarrow H C_{m-2}^{h}(A) \cdots
$$

Thus i) follows from 3.1. The natural shift isomorphism $S: B P(A) \rightarrow$ $B(P)(A)[-2]$ induces the shift isomorphism of ii). Finally, if $\mathbf{Q} \subset A$ then $H P_{m}(A) \cong H P_{m}(A[t])$ for all $m$ by $[\mathrm{K}, 4.3]$; iii) follows from Lemma 1.2 (cf. [W2, 5.3]).

\section{ACKNOWLEDGMENTS}

Geller wishes to thank the mathematics department of Rutgers University for their support, encouragement and hospitality during her year there. Weibel wishes to thank Christian Kassel for insisting that we write up the results of this paper.

\section{REFERENCES}

[And] D. W. Anderson, Relationships among K-theories, Lecture Notes in Math. \#341, SpringerVerlag, 1973.

[Bass] H. Bass, Algebraic K-theory, Benjamin, 1968.

[CE] H. Cartan and S. Eilenberg, Introduction to homological algebra, Princeton Univ. Press, 1956.

[G] T. Goodwillie, Cyclic homology, derivations, and the free loop space, Topology 24 (1985), 187-215.

[G2] __, Algebraic K-theory and cyclic homology, Annals Math. 124 (1986), 344-399.

[GW1] S. Geller and C. Weibel, $K_{1}(A, B, I)$, Reine und Angew. Math. 342 (1983), 12-34.

[GW2] _ $K(A, B, I):$ II, preprint, 1988.

[HKR] G. Hochschild, B. Kostant and A. Rosenberg, Differential forms on regular affine algebras, Trans AMS 102 (1963), 383-408.

[K] C. Kassel, Cyclic homology, comodules and mixed complexes, J. Algebra 107 (1987), 195-216.

[LQ] J.-L. Loday and D. Quillen, Cyclic homology and the Lie algebra homology of matrices, Comm. Math. Helv. 59 (1984), 565-591.

[Mac] S. MacLane, Homology, Springer-Verlag, 1963.

[Mat] H. Matsumura, Commutative algebra, Benjamin, 1970. 
[May] J. P. May, Simplicial objects in algebraic topology, Van Nostrand, 1967.

[W1] C. Weibel, KV-theory of categories, Trans. AMS 267 (1981), 621-635.

[W2] _ _ Nil K-theory maps to cyclic homology, Trans. AMS 303 (1987), 541-558.

[W3] _ Mayer-Vietoris sequences and mod p K-theory, Lecture Notes in Math. \#966, SpringerVerlag, 1982.

Department of Mathematics, Texas A \& M, College Station, Texas 77843

Department of Mathematics, Rutgers University, New Brunswick, New Jersey 08903 